\title{
Thermodynamics of the giant impact and evolution of the protolunar disk from $a b$ initio simulations
}

\author{
RAZVAN CARACAS ${ }^{1,2}$ AND SARAH STEWART ${ }^{3}$ \\ ${ }^{1}$ CNRS \\ ${ }^{2}$ University of Oslo \\ ${ }^{3} \mathrm{UC}$ Davis
}

Presenting Author: razvan.caracas@ens-lyon.fr

Impacts are highly energetic phenomena. They abound in the early stages of formation of the solar system, when they actively participated to the formation of large bodies in the protoplanetary disk. Later on, when planetesimals and embryo planets formed, impacts merged smaller bodies into the large planets that we know today. Giant impacts dominated the last phase of the planetary accretion, with some of these impacts leaving traces observable even today (planets tilts, moon, missing mantle, etc). The Earth was not spared, and its most cataclysmic event also contributed to the formation of the Moon.

Here we present the theoretical tools used to explore the thermodynamics of the formation of the protolunar disk and the subsequent condensation of this disk. We show how ab initiobased molecular dynamics simulations contribute to the determination of the stability field of melts, to the equilibrium between melts and vapor and the positioning of the critical points. Together all this information helps building the liquidvapor stability dome. Next we investigate the supercritical regime, typical of the post-impact state. We take a focused look to the transport properties, the formation of the first atmosphere, and compare the properties of the liquid state typical of magma oceans, to the super-critical state, typical of protolunar disks.

We apply this theoretical approach on pyrolite melts, as best approximants for the bulk silicate Earth. These simulations help us retrace the thermodynamic state of the protolunar disk and infer possible condensation paths for both the Earth and the moon.

RC acknowledges support from the European Research Council under EU Horizon 2020 research and innovation program (grant agreement 681818 - IMPACT) and access to supercomputing facilities via the eDARI gen6368 grants, the PRACE RA4947 grant, and the Uninet2 NN9697K grant. STS was supported by NASA grants NNX15AH54G and 80NSSC18K0828; DOE-NNSA grants DE-NA0003842 and DENA0003904. 